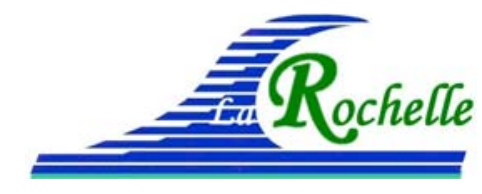

XVèmes Journées Nationales Génie Côtier - Génie Civil La Rochelle, 29 au 31 mai 2018

DOI:10.5150/jngcgc.2018.014 (C) Editions Paralia CFL

disponible en ligne - http://www.paralia.fr - available online

\title{
Comprendre le rôle des récifs barrière à Posidonia oceanica dans l'atténuation de l'hydrodynamique : Programme CANOPé
}

\author{
Anne-Eléonore PAQUIER ${ }^{1,2}$, Samuel MEULE ${ }^{1}$, Damien SOUS ${ }^{3}$, Thibault \\ OUDARD ${ }^{4}$, Philippe LARROUDE ${ }^{4}$, Élodie ROUANET ${ }^{2}$, Adrien GOUJARD ${ }^{2}$, \\ Laurence LE DIREACH ${ }^{2}$
}

1. Aix Marseille Université, CNRS, IRD, INRA, Collège de France, CEREGE, Aix-enProvence, France.

paquier@cerege.fr

2. GIS Posidonie, Institut Pythéas - Aix- Marseille Université, Campus de Luminy, OCEANOMED, Bâtiment Méditerranée, case 901, 13288 Marseille cedex 09.

3. Université de Toulon, Aix-Marseille Université, CNRS/INSU, IRD, MIO, UM 110, 83957, La Garde Cedex, France.

4. Université de Grenoble Alpes, Grenoble, INP, LEGI, 38000 Grenoble, France.

\section{Résumé :}

CANOPé est un programme pluridisciplinaire dédié à l'étude des récifs barrières de Posidonia oceanica mettant à contribution des scientifiques dont l'expertise couvrent les domaines de la cartographie, de la biologie, de l'écologie, de la géomorphologie, de l'océanographie physique et de la modélisation. Alors que le projet a pour but de comprendre l'étendue de ces monuments naturels très peu étudiés et leurs fonctions biologiques et écologiques, ce papier présente l'avancée des travaux menés pour la compréhension de la modification de l'hydrodynamique par les récifs de Posidonie. Ceci est la première étape dans la compréhension de leurs fonctions de limitation des risques d'érosion et de submersion dans un contexte de changement climatique.

Durant un événement de vent, la pression a été mesurée le long d'un transect parallèle à l'orientation des vagues et traversant un récif barrière à Posidonia oceanica à La Madrague de Giens, Parc national de Port-Cros, France. Les mesures réalisées et les premiers résultats montrant l'impact du récif sur les ondes sont présentés dans ce manuscrit.

\section{Mots-clés :}

Récifs barrières de Posidonia oceanica, Eco-hydraulique, Modification de vagues de vent, Zone critique, Nature-based defenses, Erosion côtière

\section{Introduction}

Posidonia oceanica (Linnaeus) Delile est une magnoliophyte endémique de Méditerranée qui se développe de la surface jusqu'à 20-45 $\mathrm{m}$ de profondeur et forme de larges herbiers sous-marins (MOLINIER \& PICARD, 1952 ; BOUDOURESQUE et al. 


\section{Thème 1 - Hydrodynamique côtière}

2009 ; PERGENT et al., 2012). Ces herbiers grandissent sur la base de leur propre matte (ensemble des rhizomes, racines et du sédiment piégé entre eux) qui, au cours du temps, monte vers la surface (BOUDOURESQUE \& JEUDY DE GRISSAC, 1983). Cette espèce peut édifier des récifs barrières par bio-constructions (MOLINIER \& PICARD, 1952). Ceux-ci se caractérisent par une faible profondeur au sommet et la présence d'un lagon entre le récif et la plage (BOUDOURESQUE et al. 2014, BONHOMME et al., 2015). BONHOMME et al., 2015 évaluent au nombre de 35 les sites avec des récifs barrières sur le littoral français (Corse comprise) et probablement plus d'une soixantaine dans le reste de la Méditerranée. BOUDOURESQUE et al. (2014) ont montré qu'il existait plusieurs types de récifs barrières ; typologie qui reste à compléter et à affiner.

Les travaux s'intéressant aux rôles des herbiers sous-marins ont permis de mettre en évidence les effets élémentaires des herbiers sur l'hydrodynamique: diminution des vitesses de courant dans la canopée (GAMBI et al., 1990 ; GRANATA et al., 2001), modification de la production et de la dissipation de turbulences (GAMBI et al., 1990 ; WIDDOWS et al., 2008 ; PUJOL \& NEPF, 2012), réduction de l'énergie et de la hauteur des vagues (LOWE et al., 2007 ; MANCA et al., 2012 ; PAUL \& AMOS, 2011). La capacité à atténuer les forçages hydrodynamiques est dépendante des caractéristiques biométriques comme la densité (PAUL \& AMOS, 2011 ; KOFTIS et al., 2013), l'indice de surface foliaire (PAUL et al., 2012), la flexibilité (PERALTA et al., 2008 ; PUJOL \& NEPF, 2012), le ratio de submersion (MANCA et al, 2012 ; JOHN et al, 2016), et des caractéristiques morphologiques (étendue - GAMBI et al., 1990, fragmentation - FONSECA \& KOEHL, 2006) de chaque espèce (ELGINOZ et al., 2011). À notre connaissance, seules deux études récentes se sont intéressées au rôle de la végétation en très faible profondeur (WANG et al., 2015) ou d'algues sur des récifs (POMEROY et al., 2017) et aucune n'a porté sur le rôle des récifs barrières de Posidonie sur la modification de l'hydrodynamique.

Le but du programme CANOPé est d'inventorier, de cartographier et d'améliorer la typologie des récifs barrières de Posidonie pour établir un diagnostic de leurs fonctions écologiques et comprendre comment les récifs modifient l'hydrodynamique à la côte. Ce manuscrit présente un jeu de données in situ acquis dans ce cadre et plus particulièrement la modification des vagues de vents, par ce type de structures.

\section{Méthodes}

\subsection{Sites d'étude}

L'atelier hydrodynamique du programme CANOPé porte sur les herbiers récifs de la rade de Hyères et de la Presqu'île de Giens (Parc national de Port-Cros, France). La rade de Hyères est dominée par des vents modérés à forts (maximum $17.6 \mathrm{~m} . \mathrm{s}^{-1}$ ) de Nord (N), Nord-Ouest/Nord-Nord-Ouest (NNW), Sud-Est (SE) et Est (E), les trois 


\section{XVèmes Journées Nationales Génie Côtier - Génie Civil \\ La Rochelle, 29 au 31 mai 2018}

dernières produisant les vents les plus intenses (figure 1b). Trois sites ateliers ont été choisis pour leurs différentes expositions aux forçages hydrodynamiques et leur variété de longueur de fetch (figure 1a). Le site de la Madrague, situé à l'Ouest de la presqu'île de Giens, est exposé aux vents de N à NW (fetch maximum de $5 \mathrm{~km}$ à la Madrague). Le site de la Badine, situé à l'Est de la presqu'île, est exposé à des houles générées par le vent d'E sur un fetch de $25 \mathrm{~km}$ et fortement réfractées avant de se propager dans la baie qui abrite l'herbier récif. Enfin, le site des Vieux Salins, situé au Nord de la rade de Hyères, est exposé à de rares mais forts événements générés par des vents de SE sur un fetch illimité (figure 1a).

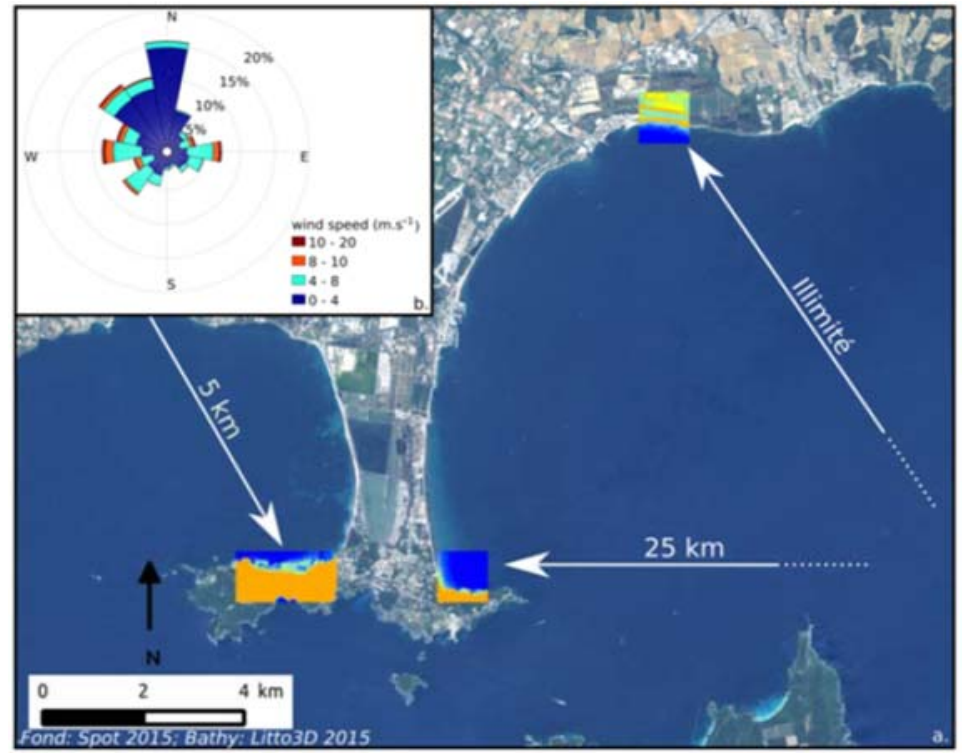

Figure 1. Sites d'étude.

Ces récifs ont tous des bathymétries complexes (zones non végétalisées aux profondeurs plus marquées, zones de fracturations de la matte, variabilité des formes du fond et des profondeurs de lagons).

\subsection{Suivis topo-bathymétriques}

Un suivi topo-bathymétrique a été réalisé sur le site de la Madrague en Octobre 2017. La partie émergée et faiblement immergée a été mesurée au GPS différentiel Trimble RTK (incluant les zones les moins profondes de l'herbier) et les zones les plus profondes au sondeur couplé à un GPS différentiel. La figure 2a présente un profil topobathymétrique à la Madrague de Giens extrait des Modèles Numériques de Terrain Litto3D ${ }^{\circledR}$. Dans les secteurs où la donnée Litto3D ${ }^{\circledR}$ est manquante, les données acquises in situ ont permis d'accroître la précision des interpolations. Le récif de la Madrague est le plus large étudié et son front est bien marqué (changement bathymétrique de $2 \mathrm{~m}$ sur $\sim 15 \mathrm{~m}$ de distance). Le relief du récif est particulièrement 


\section{Thème 1 - Hydrodynamique côtière}

accidenté (tâches sableuses, notamment à 300 et $370 \mathrm{~m}$, et nombreux accidents bathymétriques de plus petite ampleur).
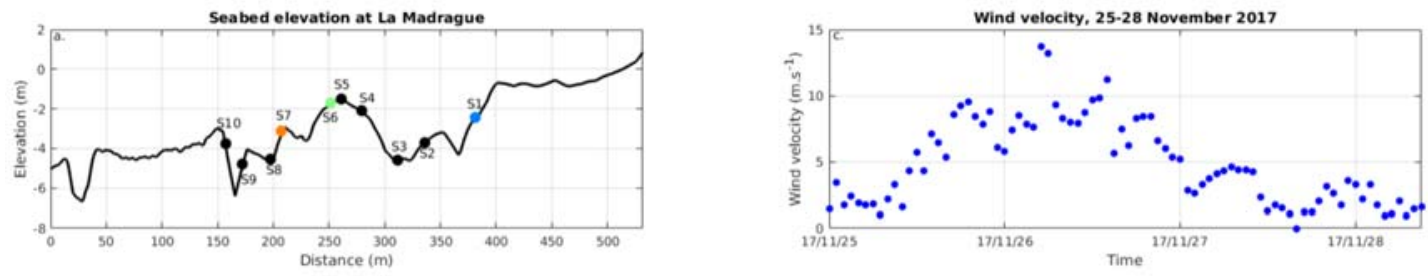

Location of the pressure sensors, 24-28 November 2017
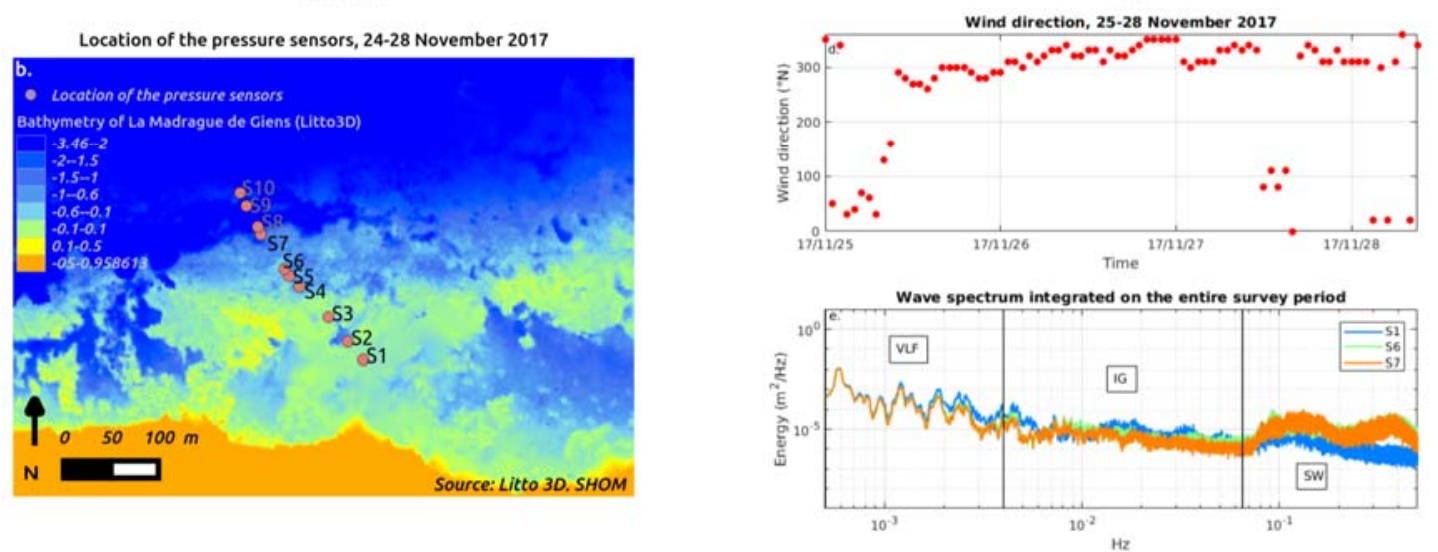

Figure 2. (a) Profil topo-bathymétrique du transect équipé de capteurs de pression et positions des capteurs. S1, S6, S7 correspondent aux données exploitées ici. (b) Bathymétrie de la Madrague (MNT Litto3D non modifié) et positions des stations de mesure. (c) Vitesse et (d) direction du vent mesurées à la B.A.N. de Hyères. (e) Spectres d'énergie des ondes calculés sur l'ensemble de la période de mesure pour S1, S6, S7.

\subsection{Suivis hydrodynamiques et traitement des données}

Un suivi hydrodynamique a été réalisé sur le site de la Madrague du 24 au 28 novembre 2017. 10 capteurs de pressions (NKE SPT10m) ont été déployés sur un transect de manière à être, a priori, dans l'axe d'incidence attendue des vagues (figures $2 \mathrm{a}-2 \mathrm{~b}$ ). Ils mesuraient la pression en continu à une fréquence de $4 \mathrm{~Hz}$.

Les données de 3 capteurs seulement seront présentées dans ce papier (S1, S6, S7, figure 3a). Les données de pression ont d'abord été corrigées de la pression atmosphérique et de la dérive des capteurs. Les séries temporelles ont ensuite été traitées par analyse spectrale pour identifier les différentes composantes des fluctuations de la surface libre: $S W$ pour les vagues (houle et mer du vent), IG pour les ondes infragravitaires et $V L F$ pour les ondes de plus basse fréquence généralement liées aux oscillations propres des bassins (LOCALETTI et al., 2017). Les VLF ont été intégrées sur l'ensemble de fréquences $\left[510^{-4} ; 4.10^{-3}\right] \mathrm{Hz}$, les IG sur l'ensemble $\left[4.10^{-3} ; 6,5.10^{-2}\right]$ $\mathrm{Hz}$ et les $\mathrm{SW}$ sur l'ensemble $\left[6,5.10^{-2} ; 5.10^{-1}\right] \mathrm{Hz}$. Les hauteurs significatives associées sont calculées sur chacun de ces ensembles. Les périodes des ondes $S W$ ont également été extraites de ces analyses spectrales (fréquences de pic, $\mathrm{T}_{\mathrm{p}}$ ). 


\section{XVèmes Journées Nationales Génie Côtier - Génie Civil \\ La Rochelle, 29 au 31 mai 2018}

\section{Premiers résultats et discussion}

Les données présentées ont été acquises lors d'un épisode de vent de NNW modéré (maximum $15 \mathrm{~m} . \mathrm{s}^{-1}$ le 26 novembre, figures 2c-2d), du 25 au 28 novembre 2017. L'analyse spectrale des signaux de pression sur l'ensemble de la période de mesure (figure 2e) montre la présence d'ondes longues $(V L F)$. On observe la présence de plusieurs pics bien définis dans la bande VLF. Ils correspondent aux modes d'oscillation propre à la rade, qui se comporte comme un bassin semi-fermé. L'énergie des $I G$ révèle les transferts d'énergie vers les basses fréquences lors du déferlement (BERTIN et al. 2018).
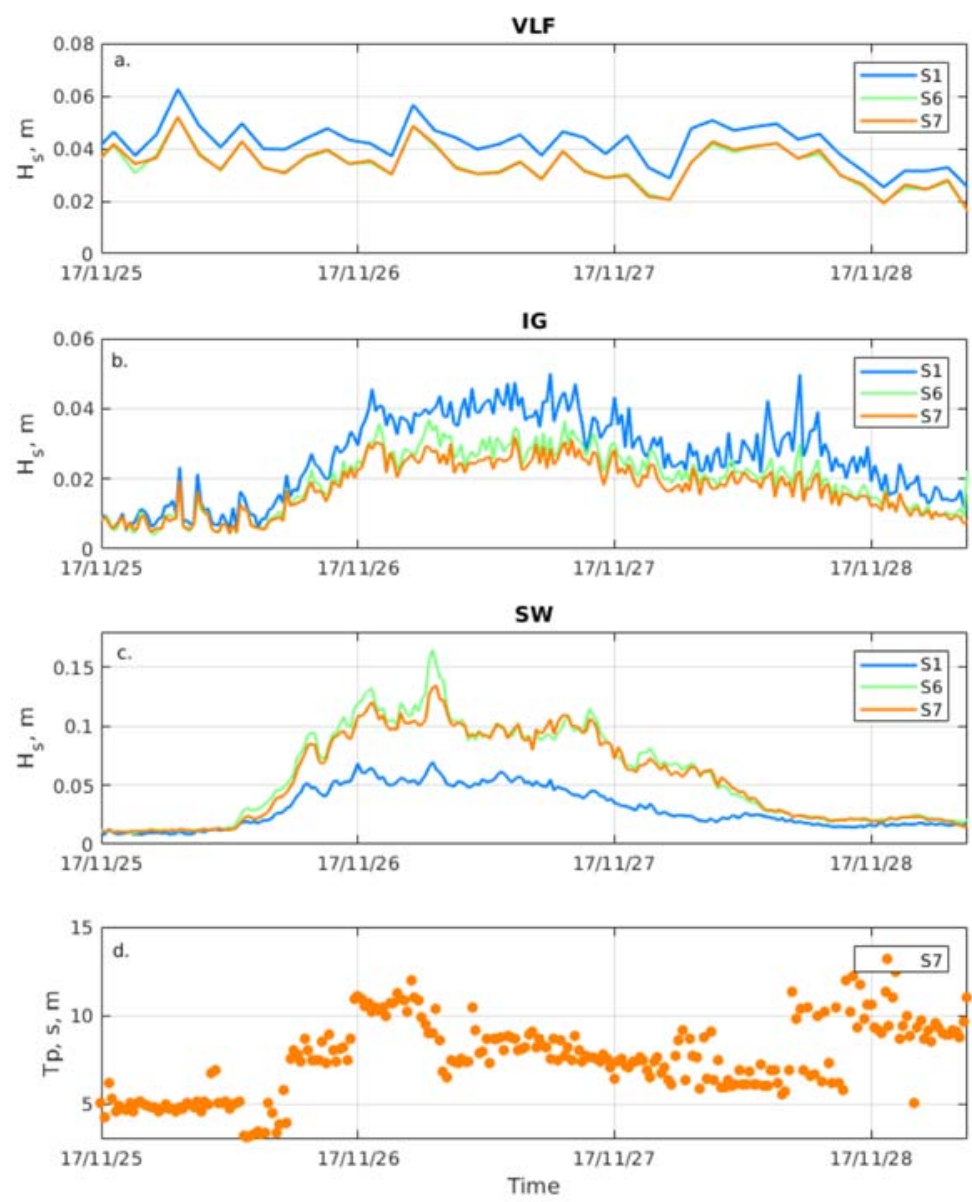

Figure 3. Hauteurs significatives des ondes VLF (a), IG (b) et SW (c) aux stations S1, S6, S7. Périodes des ondes SW (d) à la station S7.

On note que les périodes des $S W$ sont relativement longues (entre 6 et $12 \mathrm{~s}$, figure $3 \mathrm{~d}$ ), indiquant que le forçage de vague observé sur le site n'est pas généré localement. Nous supposons que la houle mesurée sur le site est donc soumise à de forts effets de réfraction avant d'arriver sur le pied du récif. Les fluctuations de VLF sont globalement 


\section{Thème 1 - Hydrodynamique côtière}

constantes dans l'espace et le temps. On observe cependant une tendance moyenne à la décroissance au cours du temps qui semble indépendante des évolutions du forçage, et une légère amplification le long du profil (figure 3a). Les hauteurs significatives des $S W$ et $I G$ sont davantage contrôlées par les conditions météo-marines (figures 3b-3c). Les transferts d'énergie le long du profil confirment les observations réalisées sur les spectres moyens (figure 3f), avec une croissance de l'énergie $I G$ et une décroissance des $S W$. Ceci est particulièrement visible entre $S 6$ et $S 1$, là où le déferlement doit être le plus marqué. Une augmentation de hauteur significative des $S W$ est aussi visible au passage du front de récif, entre S7 et S6, qui pourrait correspondre à un processus de shoaling précédant le déferlement (figure 3c).

\section{Conclusions et perspectives}

Ces résultats sont un premier pas dans la compréhension de la modification de l'hydrodynamique au-dessus d'un récif barrière à Posidonia oceanica. Les quelques conclusions qui en sont tirées sont à considérer avec prudence puisque le jeu de données étudié est très limité et concerne des ondes de très faible hauteur. Il reste nécessaire d'analyser des jeux de données plus conséquents. Les apports de simulations numériques seront également utiles pour comprendre le rôle joué par la présence des différents éléments de l'herbier dans la modification de l'hydrodynamique. Des simulations (TELEMAC 2D, Xbeach, 3D SPH) sont d'ailleurs en cours au sein de CANOPé (OUDART et al., 2018).

\section{Remerciements}

Cette étude a été réalisée avec le soutien financier de l'Agence de l'Eau RM\&C. Les données de pression atmosphérique ont été fournies par le réseau HTM-net, les données de vents par Météo-France et les données Litto3D par le SHOM.

\section{Références bibliographiques}

BERTIN X., DE BAKKER A., VAN DONGEREN A., COCO G., ANDRE G., ARDHUIN F., BONNETON P., BOUCHETTE F., CASTELLE B., CRAWFORD W. D., DAVIDSON M., DEEN M., DODET G., GUÉRIN T., INCH K., LECKLER F., MCCALL R., MULLER H., OLABARRIETA M., ROELVINK D., RUESSINK G., SOUS D., STUTZMANN E., TISSIER M., DAVIDSON, M. (2018). Infragravity waves: from driving mechanisms to impacts. Earth-Science Reviews, Vol. 177, pp 774799. https://doi.org/10.1016/j.earscirev.2018.01.002

BONHOMME D., BOUDOURESQUE C. F., ASTRUCH P., BONHOMME J., BONHOMME P., GOUJARD A., THIBAUT T. (2015). Typology of the reef formations of the Mediterranean seagrass Posidonia oceanica, and the discovery of extensive reefs 


\section{XVèmes Journées Nationales Génie Côtier - Génie Civil La Rochelle, 29 au 31 mai 2018}

in the Gulf of Hyères (Provence, Mediterranean). Scientific Report of Port-Cros national Park, Vol. 29, pp 41-73.

BOUDOURESQUE C. F., JEUDY DE GRISSAC A. (1983). L'herbier à Posidonia oceanica en Méditerranée : les interactions entre les plantes et le sédiment. J. Rech. Océanogr., Vol. 8 (2-3), pp 99-122.

BOUDOURESQUE C. F., BERNARD G., PERGENT G., SHILI A., VERLAQUE M. (2009). Regression of Mediterranean seagrasses caused by natural processes and anthropogenic disturbances and stress: a critical review. Botanica Marina, Vol. 52, pp 395-418. https://doi.org/10.1515/BOT.2009.057

BOUDOURESQUE C. F., BONHOMME D., ASTRUCH P., BONHOMME P., GOUJARD A., THIBAUT T. (2014). Insight into the typology of reef formations of the Mediterranean seagrass Posidonia oceanica. Fifth Mediterranean symposium on marine vegetation, Portorož, 27-28 October 2014, pp 58-63.

ELGINOZ E., KABDASLI M.S., TANIK A. (2011). Effects of Posidonia Oceanica Seagrass Meadows on Storm Waves. Journal of Coastal Research Special Issue, Vol. 64 (Proceedings of the 11th International Coastal Symposium), pp 373-377.

FONSECA M.S., KOEHL M. (2006). Flow in seagrass canopies: The influence of patch width. Estuarine, Coastal and Shelf Science, Vol. 67, pp 1-9. https://doi.org/10.1016/j.ecss.2005.09.018

GAMBI M. C., NOWELL A. R. M., JUMARS, P. A. (1990). Flume observations on flow dynamics in Zostera marina (eelgrass) beds. Marine Ecology Progress Series, Vol. 61, pp 159-169.

GRANATA T. C., SERRA T., COLOMER J., CASAMITJANA X., DUARTE C.M., GACIA E. (2001). Flow and particle distributions in a nearshore seagrass meadow before and after a storm. Marine Ecology Progress Series, Vol. 218, pp 95-106. https://doi.org/10.3354/meps218095

JOHN B. M., SHIRLAL K. G., RAO S., RAJASEKARAN C. (2016). Effect of artificial seagrass on wave attenuation and wave run-up. The International Journal of Ocean and Climate Systems, Vol. 7, pp 14-19. https://doi.org/10.1177/1759313115623163

KOFTIS T., PRINOS P., STRATIGAKI V. (2013). Wave damping over artificial Posidonia oceanica meadow: A large-scale experimental study. Coastal Engineering, Vol. 73, pp 71-83. https://doi.org/10.1016/j.coastaleng.2012.10.007

LOCATELLI F., SOUS D., REY V., CHEVALIER C., BOUCHETTE F., TOUBOUL J., DEVENON J.L. (2017). Wave transformation over the Ouano reef barrier, New Caledonia. Coastal Dynamics 2017, Jun 2017, Helsingor, Denmark. Proceedings of Coastal Dynamics 2017.

LOWE R. J., FALTER J. L., KOSEFF J. R., MONISMITH S. G., ATKINSON M. J. (2007). Spectral wave flow attenuation within submerged canopies: Implications for wave energy dissipation. Journal of Geophysical Research: Oceans, Vol. 112. https://doi.org/10.1029/2006JC003605 


\section{Thème 1 - Hydrodynamique côtière}

MANCA E., CÁCERES I., ALSINA J. M., STRATIGAKI V., TOWNEND I., AMOS C. L. (2012). Wave energy and wave-induced flow reduction by full-scale model Posidonia oceanica seagrass. Continental Shelf Research, Vol. 50-51, pp 100-116.

https://doi.org/10.1016/j.csr.2012.10.008

MOLINIER R., PICARD J. (1952). Recherches sur les herbiers de phanérogames marines du littoral méditerranéen français. Ann. Inst. Océanogr., Fr., Vol. 27(3), pp 157-234.

OUDART T., LARROUdÉ P., PAQUIER A. E., MEULÉ S., LEBOUTEILLER C., ROUANET E. (2018). Numerical approach for flow/vegetation interaction with 3D SPH model. Sixth International Conference on Estuaries and Coasts (ICEC-2018), August 20-23, 2018, Caen, France.

PERGENT G., BAZAIRI H., BIANCHI C. N., BOUDOURESQUE C. F., BUIA M. C., CLABAUT P., HARMELIN-VIVIEN M., MATEO M. A., MONTEFALCONE M., MORRI C., ORFANIDIS S., PERGENT-MARTINI C., SEMROUD R., SERRANO O., VERLAQUE M. (2012). Mediterranean seagrass meadows: resilience and contribution to climate change mitigation. A short summary. IUCN publ., Gland, Málaga, pp 1-40.

PAUL M., AMOS C. L. (2011). Spatial and seasonal variation in wave attenuation over Zostera noltii. Journal of Geophysical Research: Oceans, Vol. 116. https://doi.org/10.1029/2010JC006797

PAUL M., BOUMA T., AMOS C. L. (2012). Wave attenuation by submerged vegetation: combining the effect of organism traits and tidal current. Marine Ecology Progress Series, Vol. 444, pp 31-41. https://doi.org/10.3354/meps09489

PERALTA G., VAN DUREN L., MORRIS E., BOUMA T. (2008). Consequences of shoot density and stiffness for ecosystem engineering by benthic macrophytes in flow dominated areas: a hydrodynamic flume study. Marine Ecology Progress Series, Vol. 368, 103-115. https://doi.org/10.3354/meps 07574

POMEROY A. W. M., LOWE R. J., GHISALBERTI M., STORLAZZI C., SYMONDS G., ROELVINK D. (2017). Sediment transport in the presence of large reef bottom roughness. Journal of Geophysical Research: Oceans, Vol. 122. https://doi.org/10.1002/2016jc011755

PUJOL D. \& NEPF H. (2012). Breaker-generated turbulence in and above a seagrass meadow. Cont. Shelf Res. Vol. 49, pp 1-9. https://doi.org/10.1016/j.csr.2012.09.004 WANG B., GUO X., MEI C. C. (2015). Surface water waves over a shallow canopy, Journal of Fluid Mechanics, Vol. 768, pp 572-599. https://doi.org/10.1017/jfm.2015.110 WIDDOWS J., POPE N., BRINSLEY M., ASMUS H., ASMUS R. (2008). Effects of seagrass beds (Zostera noltii and Z. marina) on near-bed hydrodynamics and sediment resuspension. Marine Ecology Progress Series, Vol. 358, pp 125-136. https://doi.org/10.3354/meps07338 